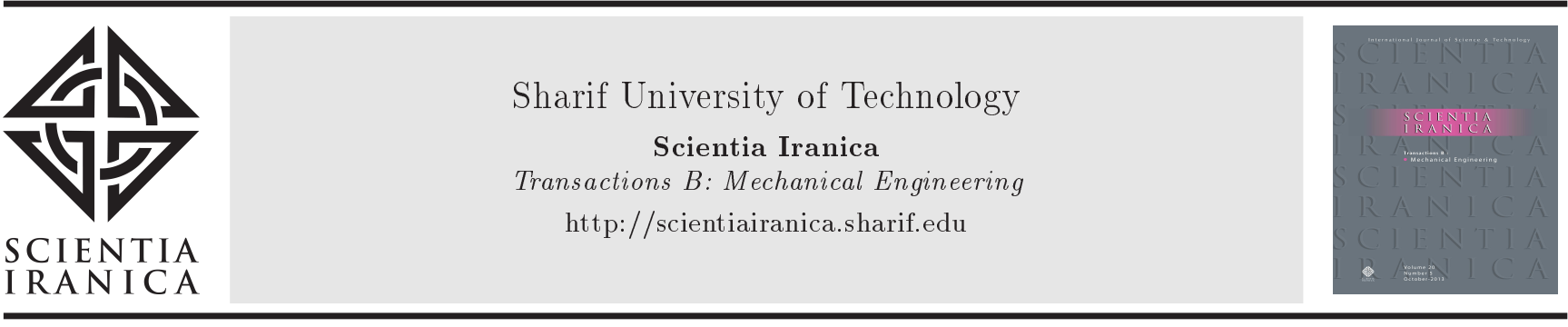

\title{
Soret and Dufour effects on doubly diffusive convection of nanofluid over a wedge in the presence of thermal radiation and suction
}

\author{
R. Md. Kasmani ${ }^{\mathrm{a}}$, S. Sivasankaran ${ }^{\mathrm{b}, *}$, M. Bhuvaneswari ${ }^{\mathrm{b}}$, A.S. Alshomrani ${ }^{\mathrm{b}}$, \\ and Z. Siric \\ a. Centre for Foundation Studies in Science, University of Malaya, Kuala Lumpur 50603, Malaysia. \\ b. Department of Mathematics, King Abdulaziz University, Jeddah 21589, Saudi Arabia. \\ c. Institute of Mathematical Sciences, University of Malaya, Kuala Lumpur 50603, Malaysia.
}

Received 21 May 2017; received in revised form 13 March 2018; accepted 15 September 2018

\section{KEYWORDS}

Soret and Dufour;

Double diffusive;

Nanoliquid;

Wedge;

Radiation.

\begin{abstract}
This paper investigates the effects of thermal radiation, Dufour, and Soret effects on doubly diffusive convective heat transfer of nanoliquid over a wedge in the presence of wall suction. The governing equations are transformed to nonlinear ordinary differential equations using similarity transformation. The resulting system is solved numerically by the fourth-order Runge-Kutta-Gill method with a shooting technique and a Newton-Raphson method. The solutions are expressed in terms of velocity, temperature, solutal concentration, and volume fraction profiles. The effects of pertinent parameters involved in the problem such as wedge angle, thermal radiation, Brownian motion, thermophoresis, Soret number, and Dufour number on the skin friction coefficient, local Nusselt number, and local Sherwood number are discussed in detail.
\end{abstract}

(C) 2019 Sharif University of Technology. All rights reserved.

\section{Introduction}

Fluid flow and energy transport characteristics of nanoliquid have received extensive attention. This attention stems from a wide range of engineering applications such as machine cooling, cooling of heat exchanging devices, electronics and transformer, improving diesel generator proficiency, improving energy transport efficiency of chillers, and cooling in nuclear reactor [1-4]. Nanoliquid is a diffusion of metallic or non-metallic nano-particles in a base liquid, resulting in the modification of the fluid properties such as viscosity, density, and thermal conductivity. Unquestionably, nanoliquids exhibit some distinctive features

*. Corresponding author. Tel.: +966 546649072

E-mail address: sd.siva@yahoo.com (S. Sivasankaran).

doi: $10.24200 /$ sci. 2018.20997 that are quite dissimilar to colloidal suspensions [46]. Numerous studies have been conducted on the modeling of convective heat transfer in nanoliquid, e.g., Buongiorno [1], Sivasankaran and Pan [2], Khorasanizadeh et al. [3], Sivasankaran et al. [4], Abdul Hakeem et al. [5], Hayat et al. [7], and Sheikholeslami and Ganji [8].

Furthermore, the problem of doubly diffusive convection saturated with nanoliquid has been extensively investigated. Bhuvaneswari et al. [9] indicated that double-diffusive effect was a subject of intensive research due to a wide range of applications in engineering and technology. These include geophysics, oceanography, isotope separation, oil-reservoirs, and mixture between gases. Kuznetsov and Nield [10] explored the doubly diffusive convection of nanoliquid past a vertical plate. The Cheng-Minkowycz problem for the doubly diffusive natural convective boundary layer flow of nanoliquid in a porous medium was 
studied by Nield and Kuznetsov [11]. They attained correlations for heat transfer data in terms of the reduced Nusselt number. They witnessed that the reduced Nusselt number increased with an increase in buoyancy ratio.

Dufour or diffusion-thermal effect is the contribution to the thermal energy flux due to concentration gradients. From another point of view, mass fluxes can be generated by temperature gradients referred to as Soret or thermo-diffusion effect [12-14]. It is well known that thermal radiation alters the temperature distribution by playing a role including controlling energy transport process, polymer processing, and nuclear reactor cooling system [15-18]. Furthermore, according to Ahmed et al. [19], that part of radiation required for designing several advanced energy convection systems that function well at high temperatures is of significance. Bhuvaneswari et al. [20] mentioned that the study of convective energy transport involved in the occurrence of thermal radiation has been brought into many scholars' attention due to its wide range of applications. Lee et al. [21] used Lie group analysis to investigate the effect of radiation on free convection flow past an inclined plate in a saturated porous medium. The effects of radiation and Hall currents on MHD flow over a plate were investigated by Das et al. [22]. Nandy and Pop [23] studied the MHD stagnation-point flow and energy transport of nanoliquid past a shrinking surface in the presence of thermal radiation. They established that the reduced Nusselt number increased with a rise in radiation and magnetic field parameters. Hayet et al. [24,25] explored the effect of thermal radiation on convective flow of boundary layer flow.

A milestone contribution in wedge flow was made by Falkner and Skan [26] who first published their boundary layer solutions. Since then, the boundary layer flow and energy transport along a wedge have been greatly developed and widely applied to many fields of geophysical and engineering, for example, in drying processes, geothermal and petroleum reservoir operations, building construction, and solar collectors. Hossain et al. [27] deliberated the forced convection non-Darcy flow over a wedge in a porous medium. They examined the effects of connective inertia, solid boundary, porous inertia, and Darcy flow resistance for fluids of Prandtl number ranging from 0.7 to 100 . Uddin and Kumar [28] investigated the influence of Hall and ion-slip effects on MHD boundary layer flow of a micro polar fluid past a wedge. They observed that the local skin friction coefficient raised by growing the wedge angle and Hall effect parameters. Yih [29] investigated the radiation effect on combined convection over a wedge in a porous medium, and found that the local Nusselt number increased with the growing radiation parameter. Watanabe et al. [30] inspected the combined convection over a wedge in the occurrence of injection/suction. Their results indicated that the skin friction and energy transport rate increased with an increase in the suction value. Kasmani et al. [31] studied the effect of chemical reaction on convective energy transport of nanoliquid over a wedge with heat generation/absorption and suction. They found that the solutal concentration declined with the growing chemical reaction parameter. Chamkha et al. [32] inspected the combined convection of nanoliquid over a wedge in a porous medium in the occurrence of thermal radiation.

Motivated by these studies, the present paper has undertaken to explore the combined effects of Dufour and Soret on doubly diffusive convective flow of nanoliquid over a wedge in the occurrence of thermal radiation and suction. In addition, the pertinent parameters of wedge angle, Brownian motion, and thermophoresis are also considered here.

\section{Mathematical formulation}

This study considers the steady, two-dimensional, incompressible, laminar flows and energy transport of nanoliquid over a wedge with thermal radiation, suction, Dufour, and Soret effects. The nanoliquid is a dilute liquid-solid mixture with an identical volume fraction of nanoparticles dispersed within the base liquid. The base liquid and nanoparticles are in thermal equilibrium. The effects of Brownian motion and thermophoresis are included for the nanoliquid. Figure 1 demonstrates the model and physical configurations. The $x$-axis is taken along the wedge surface and $y$-axis normal to the wedge surface. The flow is assumed to be in the $x$-direction. The total angle of the wedge is denoted by $\Omega=\beta \pi$, where $\beta$ is the Hartree pressure gradient. The wedge surface is presumed to be permeable so as to permit for possible wall suction, $\nu_{0}$. The thermal-diffusion (Soret) and diffusion-thermo (Dufour) effects are considered in this study [12,13,33]. Thus, in accordance with these assumptions and by employing the usual boundary layer approximations,

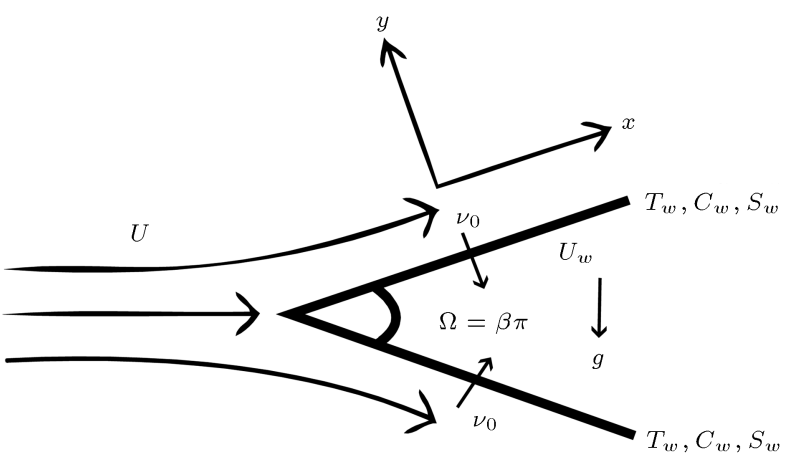

Figure 1. The physical configuration of the wedge. 
along with Boussinesq's approximation, the governing model following Kuznetsov and Nield [10] can be written as follows:

$$
\begin{aligned}
& \frac{\partial u}{\partial x}+\frac{\partial v}{\partial y}=0, \\
& u \frac{\partial u}{\partial x}+\nu \frac{\partial u}{\partial y}=\nu \frac{\partial^{2} u}{\partial y^{2}}+U \frac{d U}{d x}, \\
& u \frac{\partial T}{\partial x}+v \frac{\partial T}{\partial y} \\
& =\alpha \frac{\partial^{2} T}{\partial y^{2}}+\tau\left[D_{B} \frac{\partial S}{\partial y} \frac{\partial T}{\partial y}+\left(\frac{D_{T}}{T_{\infty}}\right)\left(\frac{\partial T}{\partial y}\right)^{2}\right] \\
& +D_{T C} \frac{\partial^{2} C}{\partial y^{2}}-\frac{1}{(\rho c)_{f}} \frac{\partial q_{r}}{\partial y} \\
& u \frac{\partial C}{\partial x}+v \frac{\partial C}{\partial y}=D_{S} \frac{\partial^{2} C}{\partial y^{2}}+D_{C T} \frac{\partial^{2} T}{\partial y^{2}}, \\
& u \frac{\partial S}{\partial x}+v \frac{\partial S}{\partial y}=D_{B} \frac{\partial^{2} S}{\partial y^{2}}+\frac{D_{T}}{T_{\infty}} \frac{\partial^{2} T}{\partial y^{2}},
\end{aligned}
$$

subject to the following boundary conditions:

$$
\begin{aligned}
& u=0, \quad v=v_{0}, \quad T=T_{w}, \quad C=C_{w}, \\
& S=S_{w}, \quad \text { at } \quad y=0, \\
& u \rightarrow U, \quad T \rightarrow T_{\infty}, \quad C \rightarrow C_{\infty}, \\
& S \rightarrow S_{\infty}, \quad \text { as } \quad y \rightarrow \infty,
\end{aligned}
$$

where $U=a x^{m}$ is the potential flow velocity, and $m=$ $\beta /(2-\beta) \geq 0$ is a wedge angle parameter. The radiative heat flux, $q_{r}=-\left(4 \sigma / 3 K^{*}\right)\left(\partial T^{4} / \partial y\right)$, is employed by utilizing the Rosseland approximation. The fluid-phase temperature differences in the flow are presumed to be sufficiently small so that $T^{4}$ can be written as a linear function of temperature by using Taylor's series about the free stream temperature, $T_{\infty}$, and disregarding the higher-order terms to yield $T^{4} \cong 4 T_{\infty}^{3} T-3 T_{\infty}^{4}$. By applying this approximation, the radiative heat flux in Eq. (3) becomes $q_{r}=-\left(16 \sigma T_{\infty}^{3} / 3 K^{*}\right)(\partial T / \partial y)$.

It is convenient to nondimensionalize the equations by introducing the following variables [31]:

$$
\begin{aligned}
& \psi=f(\eta) \sqrt{\frac{2 U \nu x}{m+1}}, \quad \eta=y \sqrt{\frac{U(m+1)}{2 \nu x}}, \\
& \theta(\eta)=\frac{T-T_{\infty}}{T_{w}-T_{\infty}}, \quad \gamma(\eta)=\frac{C-C_{\infty}}{C_{w}-C_{\infty}}, \\
& \phi(\eta)=\frac{S-S_{\infty}}{S_{w}-S_{\infty}} .
\end{aligned}
$$

The stream function $(\psi)$ is defined by $v=-\partial \psi / \partial x$ and $u=\partial \psi / \partial y$. Substituting Eq. (8) into Eqs. (2)-(7), the following non-dimensional equations can be obtained as follows $[10,31]$ :

$$
\begin{aligned}
& f^{\prime \prime \prime}+f f^{\prime \prime}+\frac{2 m}{m+1}\left(1-f^{2}\right)=0 \\
& \left(1+\frac{4}{3 R}\right) \theta^{\prime \prime}+N_{B} \phi^{\prime} \theta^{\prime}+N_{T} \theta^{2}+N_{T C} \gamma^{\prime \prime}+\operatorname{Pr} f \theta^{\prime}=0 \\
& \gamma^{\prime \prime}+\operatorname{Pr} \operatorname{Le} f \gamma^{\prime}+\operatorname{Le} N_{C T} \theta^{\prime \prime}=0 \\
& \phi^{\prime \prime}+\operatorname{Pr} \operatorname{Ln} f \phi^{\prime}+\frac{N_{T}}{N_{B}}=0
\end{aligned}
$$

with the following boundary conditions:

$$
\begin{aligned}
& f=2 F_{w} /(m+1), \quad f^{\prime}=0, \quad \theta=1, \quad \gamma=1, \\
& \phi=1, \quad \text { at } \quad \eta=0, \\
& f^{\prime} \rightarrow 1, \quad \theta \rightarrow 0, \quad \gamma \rightarrow 0, \quad \phi \rightarrow 0, \\
& \text { as } \quad \eta \rightarrow \infty,
\end{aligned}
$$

where prime indicates the partial differentiation with respect to $\eta$. The non-dimensional parameters include Prandtl number $(\operatorname{Pr}=\nu / \alpha)$, Lewis number $(\mathrm{Le}=$ $\left.\alpha / D_{S}\right)$, nanoliquid Lewis number $\left(\operatorname{Ln}=\alpha / D_{B}\right)$, radiation parameter $\left(R=K^{*} k / 4 \sigma T_{\infty}^{3}\right)$, Brownian motion $\left(N_{B}=\tau D_{B}\left(S_{w}-S_{\infty}\right) / \alpha\right)$, thermophoresis parameter $\left(N_{T}=\tau D_{T}\left(T_{w}-T_{\infty}\right) / \alpha T_{\infty}\right)$, Dufour-type parameter $\left(N_{T C}=D_{T C}\left(C_{w}-C_{\infty}\right) / \alpha\left(T_{w}-T_{\infty}\right)\right)$, suction parameter $\left(F_{w}=-v_{0} \operatorname{Re}_{x}^{1 / 2} / U\right)$, Soret-type parameter $\left(N_{C T}=D_{C T}\left(T_{w}-T_{\infty}\right) / \alpha\left(C_{w}-C_{\infty}\right)\right)$, and Reynolds number $\left(\operatorname{Re}_{x}=U x / \nu\right)$.

The local skin friction coefficient, $C_{f x}$, the local Nusselt number, $\mathrm{Nu}_{x}$, and the local Sherwood number, $\mathrm{Sh}_{x}$, can be defined as follows:

$$
\begin{aligned}
C_{f x} & =\frac{\tau_{w}}{\rho_{f} U^{2}}, \quad \mathrm{Nu}_{x}=\frac{x q_{w}}{k\left(T_{w}-T_{\infty}\right)}, \quad \text { and } \\
\mathrm{Sh}_{x} & =\frac{x q_{m}}{D_{B}\left(C_{w}-C_{\infty}\right)},
\end{aligned}
$$

where:

$$
\begin{array}{cl}
\tau_{w}=\mu\left(\frac{\partial u}{\partial y}\right)_{y=0}, & q_{w}=-k\left(\frac{\partial T}{\partial y}\right)_{y=0} \\
-\frac{4 \sigma}{3 K^{*}}\left(\frac{\partial T^{4}}{\partial y}\right)_{y=0}, & q_{m}=-D_{B}\left(\frac{\partial C}{\partial y}\right)_{y=0},
\end{array}
$$

are shear stress and heat and mass fluxes, respectively. By employing the variables in Eq. (8), the local skin 
friction and Nusselt and Sherwood numbers can be written respectively as follows:

$$
\begin{aligned}
& C_{f x} \sqrt{\operatorname{Re}_{x}}=f^{\prime \prime}(0) \sqrt{\frac{m+1}{2}}, \\
& \mathrm{Nu}_{x}\left(\operatorname{Re}_{x}\right)^{-1 / 2}=-\theta^{\prime}(0)\left(1+\frac{4}{3 R}\right) \sqrt{\frac{m+1}{2}}, \\
& \operatorname{Sh}_{x}\left(\operatorname{Re}_{x}\right)^{-1 / 2}=-\gamma^{\prime}(0) \sqrt{\frac{m+1}{2}} .
\end{aligned}
$$

\section{Numerical method}

The nonlinear ordinary differential equations (9)-(12) subject to the boundary conditions (13) and (14) are numerically solved by the fourth-order Runge-KuttaGill method combined with the shooting and NewtonRaphson methods. The complete method for the solution can be found in our recent paper [33].

The justification of the in-house code is a key factor in mathematical simulation. Therefore, an inspection of the present outcomes against the existing results for validating the correctness of the current code has been concluded. Table 1 shows the comparison of the current results for $f^{\prime \prime}(0)$ and $-\theta(0)$ and those obtained by Watanabe et al. [30] for several values of wedge angle $(m)$ when $\operatorname{Pr}=0.73$. Good agreement between the results is observed, as indicated in Table 1. In this way, our mathematical calculations are indeed verified.

\section{Results and discussion}

The controlling parameters and the range of values in the study are the wedge angle parameter $(0<$ $m \leq 0.5)$, suction parameter $\left(0 \leq F_{w} \leq 2\right)$, thermal radiation $(0<R \leq 4)$, Brownian motion parameter $\left(0<N_{B} \leq 0.6\right)$, thermophoresis parameter $(0<$ $\left.N_{T}<1\right)$, Soret-type parameter $\left(0 \leq N_{C T} \leq 1\right)$, and Dufour effect $\left(0 \leq N_{T C}<0.5\right)$. Figures $2-8$ display the effects of all thermo-physical parameters on dimensionless flow, thermal and solutal fields, local skin-friction, and Sherwood and Nusselt numbers.

Figure 2(a) and (b) show the impact of wedge angle parameter $(m)$ and suction parameter $\left(F_{w}\right)$, respectively. Both figures show that the velocity of the

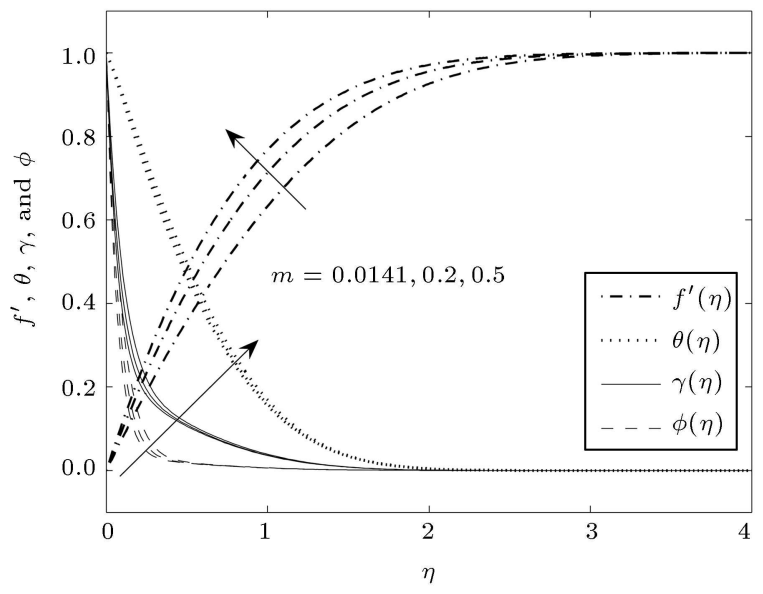

(a)

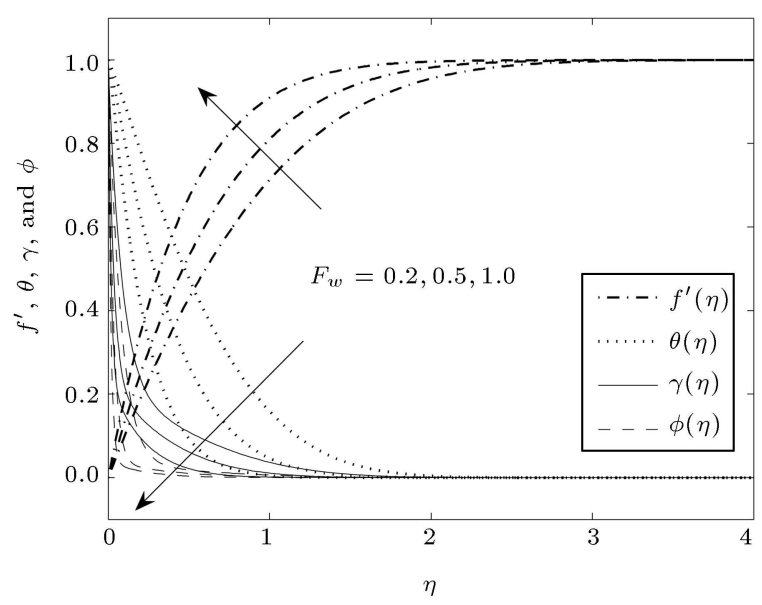

(b)

Figure 2. The velocity, temperature, solutal concentration, and volume fraction profiles with $R=1$, $N_{B}=0.2, N_{T}=0.1, N_{C T}=0.5, N_{T C}=0.1$, Le $=5$, and $\mathrm{Ln}=6$ for different values of $(\mathrm{a}) m$ when $F_{w}=0.2$ and (b) $F_{w}$ when $m=0.2$.

Table 1. Comparison of $f^{\prime \prime}(0)$ and $-\theta^{\prime}(0)$ for various values of $m$ when $\operatorname{Pr}=0.73$ with. $F_{w}=R=N_{B}=N_{T}=N_{C T}=$ $N_{T C}=\mathrm{Le}=\mathrm{Ln}=0$.

\begin{tabular}{cccccc}
\hline & \multicolumn{2}{c}{$\boldsymbol{f}^{\prime \prime}(\mathbf{0})$} & & \multicolumn{2}{c}{$-\boldsymbol{\theta}^{\prime}(\mathbf{0})$} \\
\cline { 2 - 3 } \cline { 5 - 6 } $\boldsymbol{m}$ & Watanabe et al. $[\mathbf{3 0}]$ & Present & & Watanabe et al. [30] & Present \\
\hline 0 & 0.46960 & 0.46960 & & 0.42015 & 0.42016 \\
0.0435 & 0.56898 & 0.56898 & & 0.43548 & 0.43548 \\
0.0909 & 0.65498 & 0.65498 & & 0.44730 & 0.44730 \\
0.1429 & 0.73200 & 0.73200 & & 0.45693 & 0.45694 \\
0.2000 & 0.80213 & 0.80213 & & 0.46503 & 0.46503 \\
0.3333 & 0.92765 & 0.92765 & & 0.47814 & 0.47814 \\
\hline
\end{tabular}


liquid increases with an increase in $m$ and $F_{w}$. That is, the velocity profile goes closer to the wedge surface and the hydrodynamic boundary layer diminishes for higher values of $m$ and $F_{w}$. It can be understood from Figure 2(a) that the rise of wedge angle causes an increase in thermal and solutal fields. Furthermore, Figure 2(b) shows that the solutal, temperature, and nanoparticle volume fraction profiles diminish when the suction parameter increases. This situation results in an increase in the sheer stress on the wedge surface. Moreover, it is observed that the imposition of suction on the wedge surface diminishes the region of viscous domination close to the wall, leading to a decrease in the liquid's solutal, temperature, and nanoparticle volume fraction distributions.

The influence of thermal radiation $(R)$ on temperature and volume fraction profiles is depicted in Figure 3(a). A growth in the thermal radiation leads to

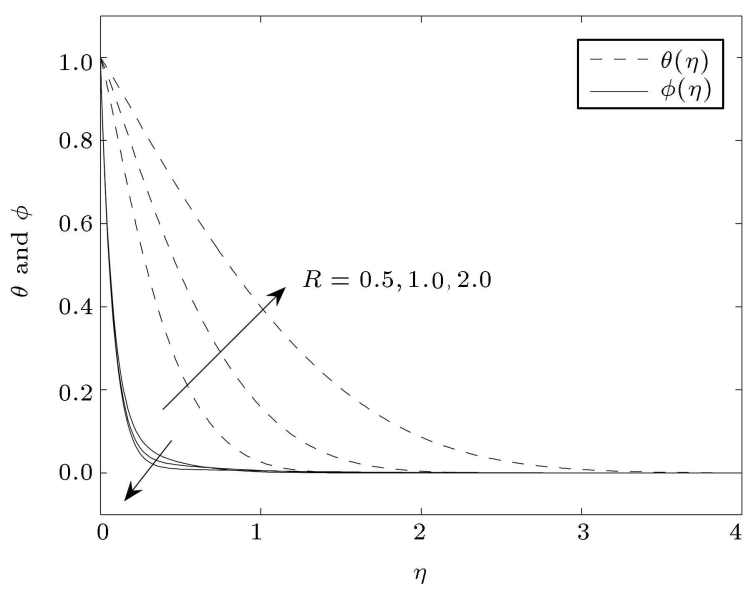

(a)

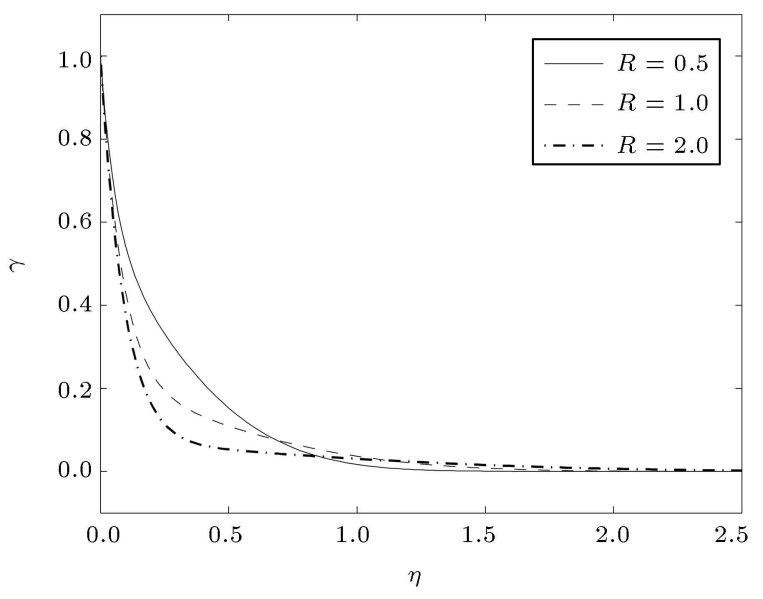

(b)

Figure 3. The influence of $R$ on (a) temperature and nanoparticle volume fraction profiles and (b) solutal concentration profile when $m=0.2, F_{w}=0.2, N_{B}=0.2$, $N_{T}=0.1, N_{C T}=0.5, N_{T C}=0.1$, Le $=5$, and $\operatorname{Ln}=6$. an increase in nanoliquid temperature. Hence, this will enhance the temperature at each point away from the wedge surface. The higher value of radiation parameter implies greater surface heat flux. This situation is accompanied by a growth in thermal boundary layer and the convection effect. In addition, Figure 3(a) shows that the nanoparticle volume fraction decreases upon increasing $R$. The effect of $\phi$ with $R$ is insignificant compared to $\theta$ on $R$. Even though the parameter $R$ is not involved in the nanoparticle volume fraction equation, there is a small change in the $\phi$ profile. Figure 3(b) displays the solutal field for various values of thermal radiation. It is witnessed that the solutal concentration distribution decreases with the rising radiation parameter.

The random movement of nanoparticles within the base liquid is called Brownian motion. The influence of Brownian motion $\left(N_{B}\right)$ is shown in Figure 4(a). It can be seen that the fluid temperature is found to increase with a growth in the Brownian motion parameter. This means that the thickness of thermal boundary layer increases as $N_{B}$ grows, which eventually enhances the temperature. The increase of the temperature distribution results from incessant collisions among the nanoparticles and the molecules of the water. In Figure 4(a), we detected that the solutal concentration and nanoparticle volume fraction decrease with a rise in Brownian motion parameter. A rise in $N_{B}$ represents a growth in the intermolecular space of nanoparticles. Thus, this situation results in lowering the nanoparticle volume fraction distribution and the nanoliquid concentration.

Figure 4(b) presents the distribution of temperature, solutal concentration, and nanoparticle volume fraction against $\eta$ for several values of thermophoresis parameter $\left(N_{T}\right)$. Thermophoresis is referred to as the transport of the nanoparticles in base fluid due to the temperature gradient under the influence of the thermophoretic force. It is witnessed that a rise in $N_{T}$ leads to an increase in both liquid temperature and nanoparticle volume fraction. This phenomenon shows that the nanoparticles' molecules carry high kinetic energy that result in a net force on the particles. These enhancements are subject to the nanoparticles of high thermal conductivity being driven away from the hot surface to the quiescent nanoliquid. This net force is called thermophoretic force. In addition, according to Figure 4(b), the solutal concentration decreases as the value of $N_{T}$ increases. This specifies that the thermophoresis parameter works to increase the volume fraction and thermal boundary layer thickness and diminishes solutal boundary layer thickness.

Figure 4(c) depicts the temperature, solutal concentration, and nanoparticle volume fraction profiles for various values of Soret-type parameter $\left(N_{C T}\right)$. It 


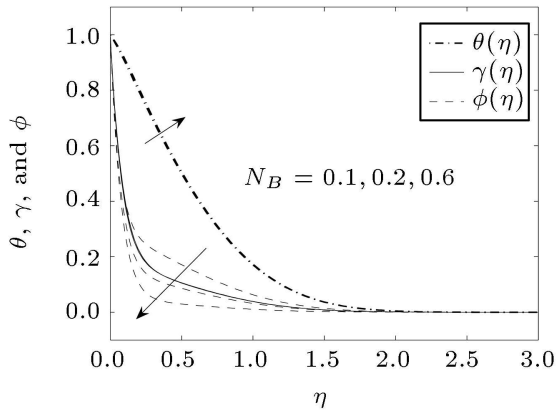

(a)

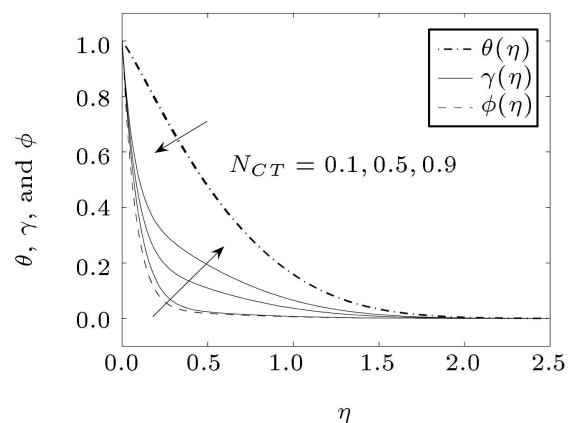

(c)

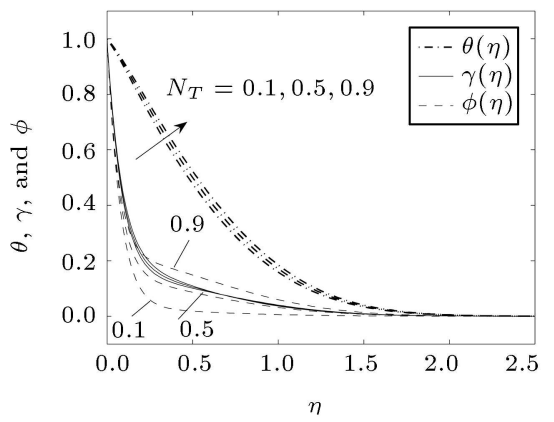

(b)

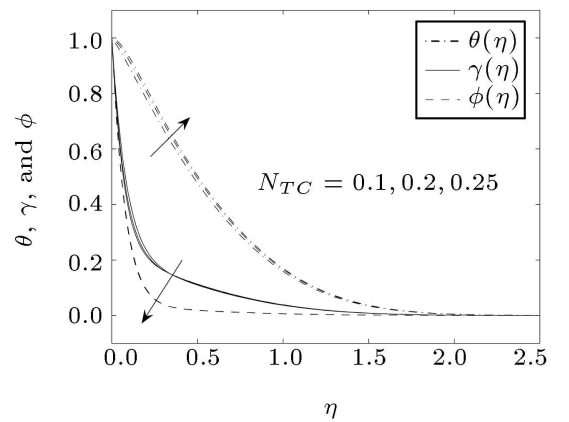

(d)

Figure 4. The distribution of $\theta, \gamma$, and $\phi$ with $m=0.2, F_{w}=0.2, R=1$, Le $=5$, and $\mathrm{Ln}=6$ for different values of (a) $N_{B}$ when $N_{T}=0.1, N_{C T}=0.5, N_{T C}=0.1$, (b) $N_{T}$ when $N_{B}=0.2, N_{C T}=0.5, N_{T C}=0.1$, (c) $N_{C T}$ when $N_{B}=0.2$, $N_{T}=0.1, N_{T C}=0.1$, and (d) $N_{T C}$ when $N_{B}=0.2, N_{T}=0.1, N_{C T}=0.5$.

is detected that a rise in the Soret-type parameter leads to a decrease in the thermal fields. The solutal concentration and nanoparticle volume fraction increase as the value of $N_{C T}$ grows. In Figure 4(d), the temperature distribution of the fluid rises when the value of $N_{C T}$ rises, which in turn leads to a decrease in nanoparticle volume fraction and the lowering of the concentration of species.

The influence of local skin friction versus suction parameter $\left(F_{w}\right)$ for several values of wedge angle $(m)$ is shown in Figure 5(a). It is found that a rise in wedge angle parameter leads to an approximate growth in the local skin friction when $F_{w}<1.4$. The opposite behavior is detected for $F_{w}>1.4$. Figure 5(b) exhibits the variation of local Nusselt number for various values of $m$. It can be seen that the value of local Nusselt number shows different behavioral patterns before and after certain points of $F_{w}$ as the wedge angle parameter rises, particularly when $F_{w}=0.45$. Before $F_{w} \approx 0.5$, the local Nusselt number rises and, after this point, it decreases. The variation of local Sherwood number for various values of $m$ is displayed in Figure $5(\mathrm{c})$. The local Sherwood number decays as the wedge angle parameter rises with a rise in suction parameter. The local mass transfer rate vanishes when the suction parameter approaches zero (i.e., $F_{w} \rightarrow 0$ ).

The variations of local Sherwood and Nusselt numbers are plotted against $N_{B}$ in Figure $6(\mathrm{a})$ and (b), respectively, for various values of thermal radiation $(R)$. It is observed that an increase in Brownian motion parameter obviously decreases the local Nusselt number, whereas the reverse effect is seen in the local Sherwood number. According to Figure 6, both the local Nusselt and Sherwood numbers are enhanced upon an increase in the value of thermal radiation. The impacts of Brownian motion and thermophoresis parameters on local Nusselt and Sherwood numbers are illustrated in Figure $7(\mathrm{a})$ and (b), respectively. The local Nusselt number diminishes as both the Brownian motion and thermophoresis parameters rise. Meanwhile, the local Sherwood number increases as $N_{B}$ and $N_{T}$ increase. This is due to the fact that an increase in energy exchange rate causes an enhancement in the random movement of nanoparticles and promotes heat conduction.

The impacts of local Nusselt and Sherwood numbers are plotted in Figure 8(a) and (b), respectively, against $N_{C T}$ for various values of Dufour-type parameter $\left(N_{T C}\right)$. The graphs show that the local Nusselt number decays as the value of $N_{T C}$ rises, while a converse tendency is detected for the local Sherwood number. Figure 8(b) indicates that the local Sherwood number increases as the Dufour-type parameter grows. According to Figure 8(a), the local Nusselt number increases upon an increase in the Soret-type parameter, which provides additional mass diffusion. Thus, the 


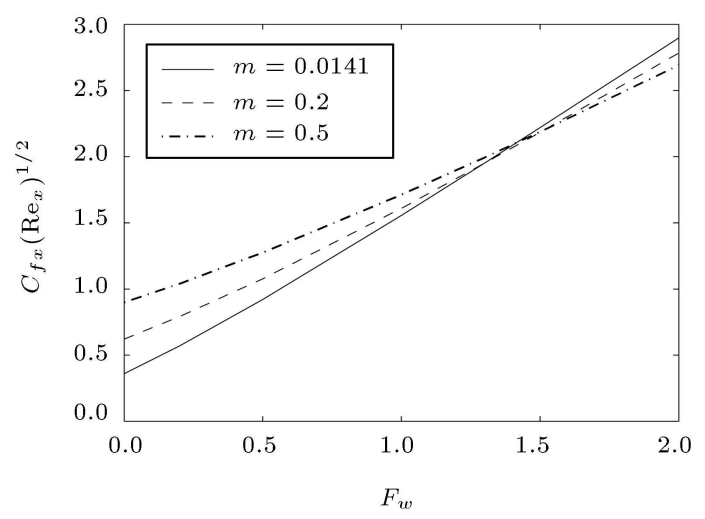

(a)

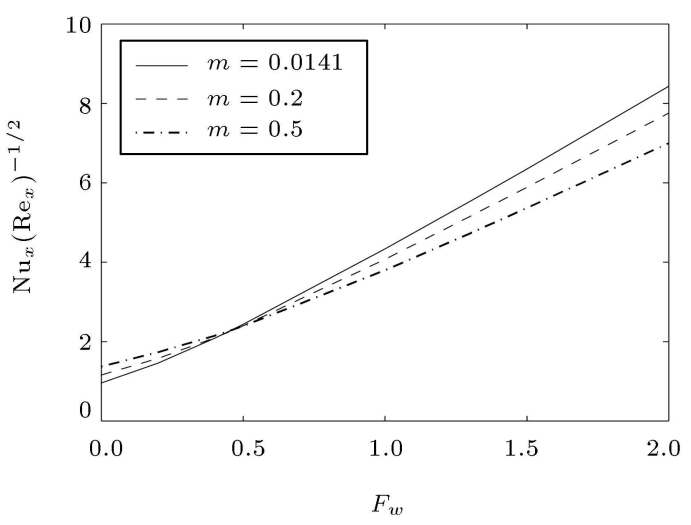

(b)

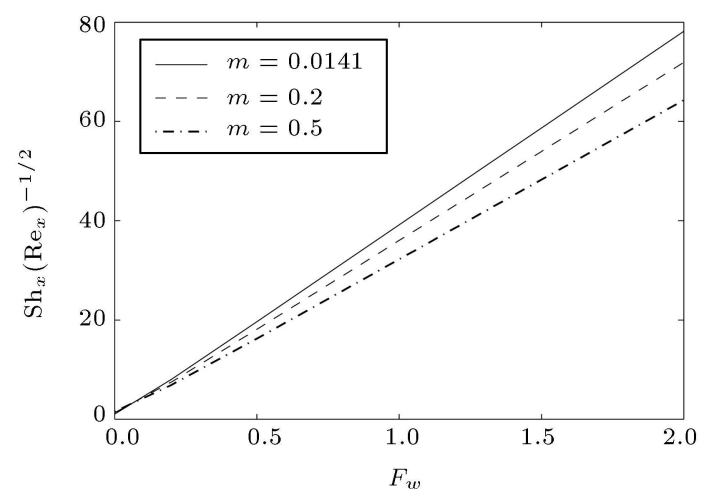

(c)

Figure 5. The variations of (a) local skin friction coefficient, (b) local Nusselt number, and (c) local Sherwood number against $F_{w}$ for different values of $m$ when $R=1, N_{B}=0.2, N_{T}=0.1, N_{C T}=0.5, N_{T C}=0.1$, $\mathrm{Le}=5$, and $\operatorname{Ln}=6$.

local Sherwood number reduces as the value of $N_{C T}$ rises. According to the comparison of these Figure 8(a) and (b), both parameters offer contradictory tendencies with respect to local mass and heat transfer rates.

\section{Conclusions}

In this work, the impacts of thermal radiation, Dufour,

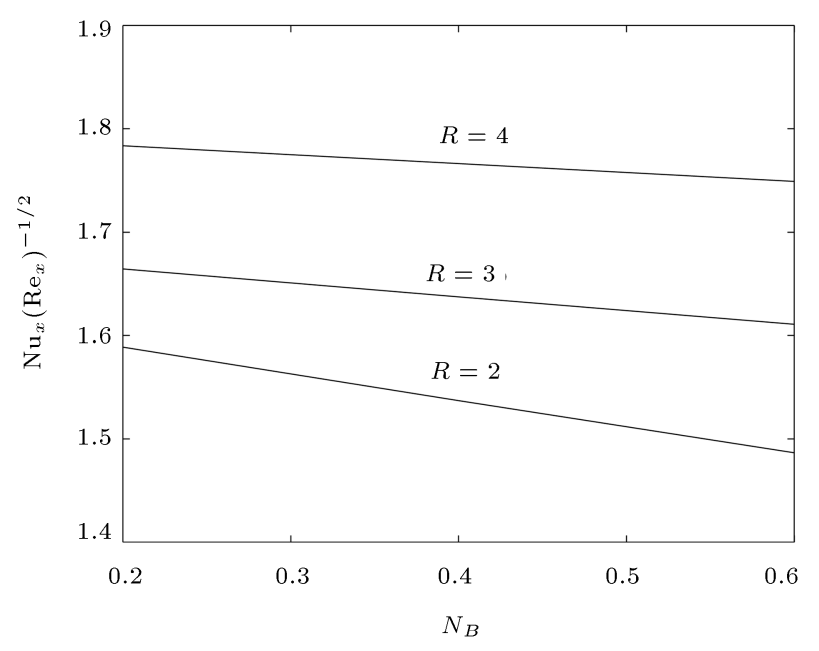

(a)

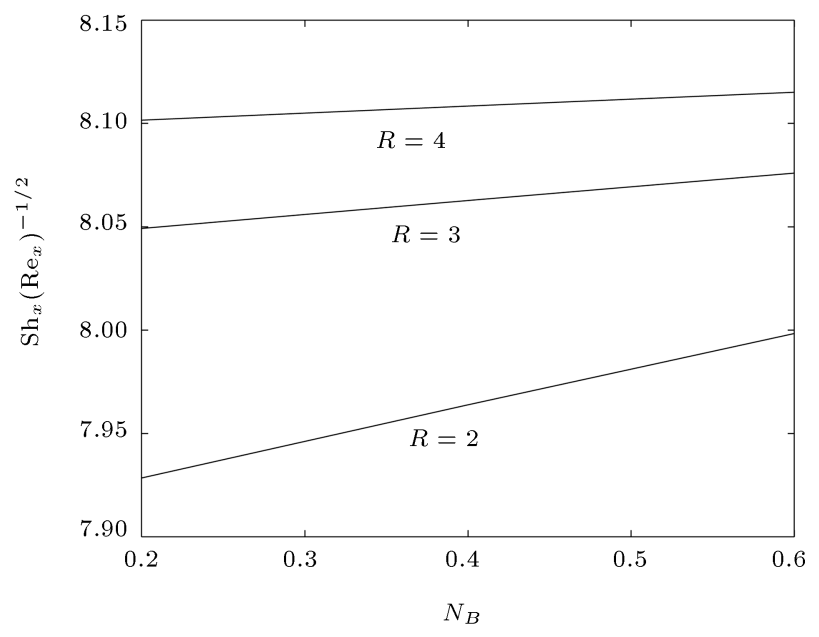

(b)

Figure 6. The variations of (a) local Nusselt number and (b) local Sherwood number against $N_{B}$ for different values of $R$ when $m=0.2, F_{w}=0.2, N_{T}=0.1, N_{C T}=0.5$, $N_{T C}=0.1, \mathrm{Le}=5$ and $\mathrm{Ln}=6$.

and Soret on the doubly diffusive flow of nanoliquid over a wedge in the presence of suction are numerically investigated. The effects of wedge angle, thermal radiation, Brownian motion, thermophoresis, Soret, and Dufour parameters are inspected. The results are stated as follows:

- The velocity raises upon increasing the values of wedge angle and suction parameters;

- The temperature distribution is enhanced as the values of wedge angle, radiation, Brownian motion, and thermophoresis parameters increase. It decays when suction and Soret-type parameters increase;

- The solutal concentration increases when the wedge angle and Soret-type parameter increase, whereas 


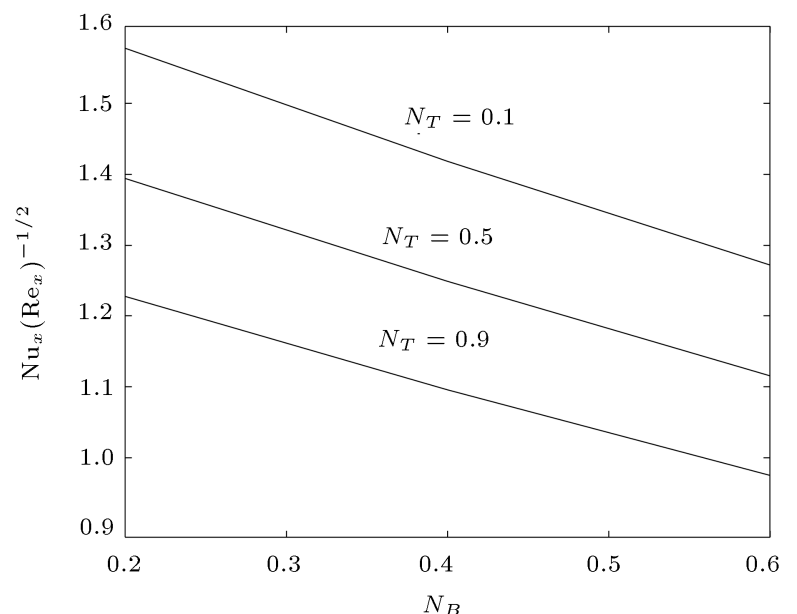

(a)

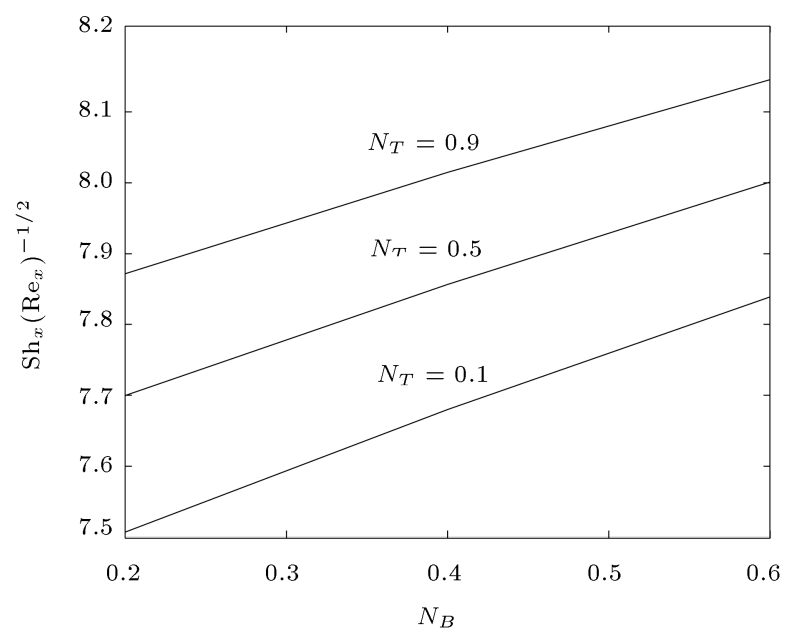

(b)

Figure 7. The variations of (a) local Nusselt number and (b) local Sherwood number against $N_{B}$ for different values of $N_{T}$ when $m=0.2, F_{w}=0.2, R=1, N_{C T}=0.5$, $N_{T C}=0.1, \operatorname{Le}=5$, and $\operatorname{Ln}=6$.

it declines with an increase in suction, radiation, Brownian motion, and thermophoresis parameters;

- The local skin friction increases upon the growth of the value of wedge angle for and $F_{w}<1.4$ decays for $F_{w}>1.4$;

- The local Nusselt number augments as wedge angle rises when $F_{w}<0.5$, and it diminishes for $F_{w}>$ 0.5. The local Nusselt number is enhanced as the effect of radiation and Soret-type parameter increase; in addition, it reduces as Brownian motion and thermophoresis parameter increase;

- The local Sherwood number decreases with an increase in the wedge angle and Soret-type parameters. Meanwhile, it increases upon increasing suction, radiation, Brownian motion, and thermophoresis parameters.

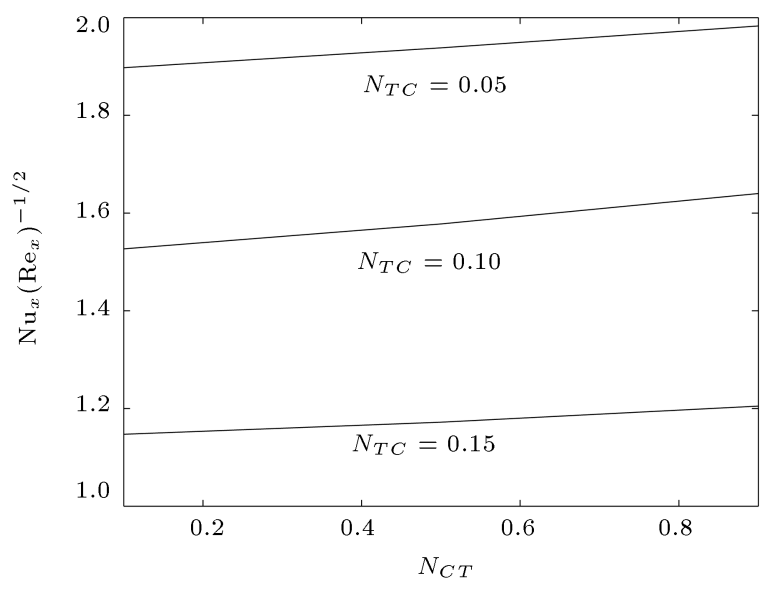

(a)

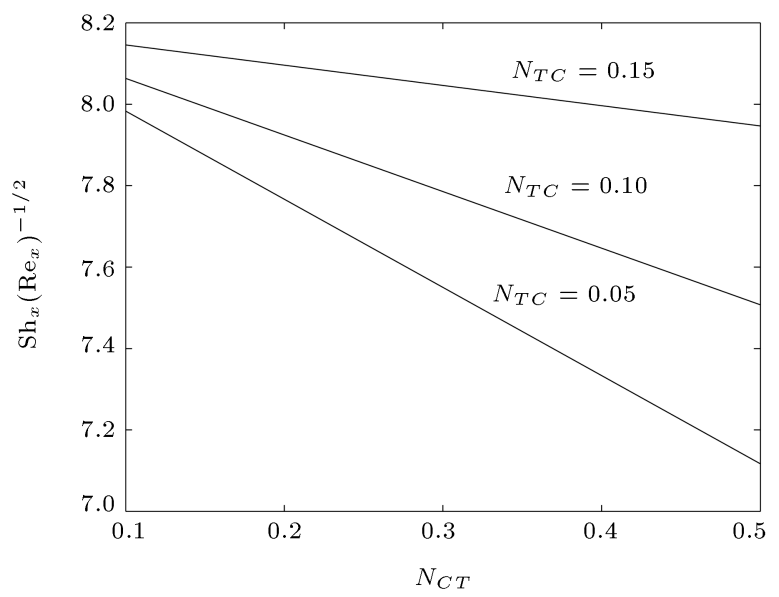

(b)

Figure 8. The variations of (a) local Nusselt number and (b) local Sherwood number against $N_{C T}$ for different value of $N_{T C}$ when $m=0.2, F_{w}=0.2, R=1, N_{B}=0.2$, $N_{T}=0.1, \mathrm{Le}=5$, and $\mathrm{Ln}=6$.

\section{References}

1. Buongiorno, J. "Convective transport in nanofluids", ASME J. Heat Transfer, 128, pp. 240-250 (2006).

2. Sivasankaran, S. and Pan, K.L. "Natural convection of nanofluids in a cavity with non-uniform temperature distributions on side walls", Numer. Heat Transfer A, 65, pp. 247-268 (2014).

3. Khorasanizadeh, H., Amani, J., and Nikfar, M. "Effect of Brownian and thermophoretic diffusions of nanoparticles on non-equilibrium heat conduction in a nanofluid layer with periodic heat flux", Sci. Iran. F, 19(6), pp. 1996-2003 (2012).

4. Sivasankaran, S., Aasaithambi, T., and Rajan, S. "Natural convection of nanofluids in a cavity with linearly varying wall temperature", Maejo Int. J. Sci. Tech., 4, pp. 468-482 (2010). 
5. Abdul Hakeem, A.K., Vishnu Ganesh, N., and Ganga, B. "Heat transfer of non-Darcy MHD flow of a nanofluid over a stretching/shrinking surface in a thermally stratified medium with second order slip model", Scientia Iranica B, 22(6), pp. 2766-2784 (2015).

6. Mollamahdi, M., Abbaszadeh, M., and Sheikhzadeh, G.A. "Analytical study of $\mathrm{Al}_{2} \mathrm{O}_{3}-\mathrm{Cu}$ /water micropolar hybrid nanofluid in a porous channel with expanding/contracting walls in the presence of magnetic field", Scientia Iranica B, 25(1), pp. 208-220 (2018).

7. Hayat, T., Ullah, I., Alsaedi, A., and Ahmad, B. "Modeling tangent hyperbolic nanoliquid flow with heat and mass flux conditions", Eur. Phys. J. Plus, 132, p. 112 (2017).

8. Sheikholeslami, M. and Ganji, D.D. "Magnetohydrodynamic flow in a permeable channel filled with nanofluid", Scientia Iranica B, 21(1), pp. 203-212 (2014).

9. Bhuvaneswari, M., Sivasankaran, S., and Kim, Y.J. "Numerical study on double diffusive mixed convection with a Soret effect in a two-sided lid-driven cavity", Numer. Heat Transfer A, 59, pp. 543-560 (2011).

10. Kuznetsov, A.V. and Nield, D.A. "Double-diffusive natural convective boundary-layer flow of a nanofluid past a vertical plate", Int. J. Thermal Sci., 50, pp. 712-717 (2011).

11. Nield, D.A. and Kuznetsov, A.V. "The ChengMinkowycz problem for the double-diffusive natural convective boundary layer flow in a porous medium saturated by a nanofluid", Int. J. Heat Mass Transfer, 54, pp. 374-378 (2011).

12. Niranjan, H., Sivasankaran, S., and Bhuvaneswari, M. "Chemical reaction, Soret and Dufour effects on MHD mixed convection stagnation point flow with radiation and slip condition", Scientia Iranica B, 24(2), pp. 698706 (2017).

13. Eswaramoorthi, S., Bhuvaneswari, M., Sivasankaran, S., and Rajan, S. "Soret and Dufour effects on viscoelastic boundary layer flow over a stretching surface with convective boundary condition with radiation and chemical reaction", Scientia Iranica B, 23(6), pp. 2575-2586 (2016).

14. Pal, D. and Mondal, H. "Soret-Dufour effects on hydromagnetic non-Darcy convective-radiative heat and mass transfer over a stretching sheet in porous medium with viscous dissipation and Ohmic heating", J. Appl. Fluid Mech., 7, pp. 513-523 (2014).

15. Hayat, T., Ullah, I., Muhammad, T., and Alsaedi, A. "Radiative three-dimensional flow with Soret and Dufour effects", Int. J. Mech. Sci., 133, pp. 829-837 (2017).

16. Abdul Hakeem, A.K., Govindaraju, M., Ganga, B. and Kayalvizhi, M. "Second law analysis for radiative MHD slip flow of a nanofluid over a stretching sheet with nonuniform heat source effect", Scientia Iranica $B, \mathbf{2 3}(3)$, pp. 1524-1538 (2016).

17. Ramly, N.A., Sivasankaran, S., and Noor, N.F.M. "Zero and nonzero normal fluxes of thermal radiative boundary layer flow of nanofluid over a radially stretched surface", Scientia Iranica B, 24(6), pp. 28952903 (2017).

18. Ghaffarpasand, O. "Natural convection and entropy generation of ultrafine atmospheric aerosols in the presence of hydrodynamic partial slip and thermal radiation due to solar energy", Scientia Iranica $B$, 24(3), pp. 1686-1705 (2017).

19. Ahmed, S.E., Hussein, A.K., Mohammed, H.A., Adegun, I.K., Zhang, X., Kolsi, L., Hasanpour, A., and Sivasankaran, S. "Viscous dissipation and radiation effects on MHD natural convection in a square enclosure filled with a porous medium", Nucl. Eng. Des., 266, pp. 34-42 (2014).

20. Bhuvaneswari, M., Sivasankaran, S., and Kim, Y.J. "Lie group analysis of radiation natural convection flow over an inclined surface in a porous medium with internal heat generation", J. Porous Media, 12, pp. 1155-1164 (2012).

21. Lee, J., Kandaswamy, P., Bhuvaneswari, M., and Sivasankaran, S. "Lie group analysis of radiation natural convection heat transfer past an inclined porous surface", J. Mech. Sci. Tech., 22, pp. 1779-1784 (2008).

22. Das, S., Guchhait, S.K., and Jana, R.N. "Effects of Hall currents and radiation on unsteady MHD flow past a heated moving vertical plate", J. Appl. Fluid Mech., 7, pp. 683-692 (2014).

23. Nandy, S.K. and Pop, I. "Effects of magnetic field and thermal radiation on stagnation flow and heat transfer of nanofluid over a shrinking surface", Int. Comm. Heat Mass Transfer, 53, pp. 50-55 (2014).

24. Hayat, T., Ullah, I., Alsaedi A., and Ahmad, B. "Radiative flow of Carreau liquid in presence of Newtonian heating and chemical reaction", Results Phys., 7, pp. 715-722 (2017).

25. Hayat, T., Ullah, I., Ahmad, B., and Alsaedi A. "MHD mixed convection flow of third grade liquid subject to non-linear thermal radiation and convective condition", Results Phys., 7, pp. 2804-2811 (2017).

26. Falkner, V.M. and Skan, S.W. "Some approximate solutions of the boundary-layer equations", Phil. Mag., 12, pp. 865-896 (1931).

27. Hossain, M.A., Banu, N., and Nakayama, A. "NonDarcy forced convection boundary layer flow over a wedge embedded in a saturated porous medium", Numer. Heat Transfer A, 26, pp. 399-414 (1994).

28. Uddin, Z and Kumar, M. "Hall and ion-slip effect on MHD boundary layer flow of a micro polar fluid past a wedge", Sci. Iran. B, 20(3), pp. 467-476 (2013). 
29. Yih, K.A. "Radiation effect on mixed convection over an isothermal wedge in porous media: The entire regime", Heat Transfer Eng., 22, pp. 26-32 (2001).

30. Watanabe, T., Funazaki, K., and Taniguchi, H. "Theoretical analysis on mixed convection boundary layer flow over a wedge with uniform suction or injection", Acta Mechanica, 105, pp. 133-141 (1994).

31. Kasmani, R.M., Sivasankaran, S., and Siri, Z. "Effect of chemical reaction on convective heat transfer of boundary layer flow in nanofluid over a wedge with heat generation/absorption and suction", J. Appl. Fluid Mech., 9(1), pp. 379-388 (2016).

32. Chamkha, A.J., Abbasbandy, S., Rashad, A.M., and Vajravelu, K. "Radiation effects on mixed convection over a wedge embedded in a porous medium filled with a nanofluid", Transport Porous Med., 91, pp. 261-279 (2012).

33. Md Kasmani, R., Sivasankaran, S., Bhuvaneswari, M., and Hussein, A.K. "Analytical and numerical study on convection of nanofluid past a moving wedge with Soret and Dufour effects", Int. J. Numerical Methods Heat \& Fluid Flow, 27(10), pp. 2333-2354 (2017).

\section{Biographies}

Ruhaila Md Kasmani is a Senior Lecturer of Mathematics at Centre for Foundation Studies at University of Malaya, Malaysia. She obtained PhD in Mathematics from the University of Malaya and completed her Master's Degree of Science with Honours at Universiti Teknologi Malaysia. Her research interests lie in the area of boundary layer flow, numerical fluid dynamics, and numerical methods.

Sivasankaran Sivanandam received his MSc, MPhil, and $\mathrm{PhD}$ degrees from Bharathiar University, India. Afterwards, he received Post-Doctoral Fellowship from
National Cheng Kung University, Taiwan, and National Taiwan University, Taiwan. He was a Research Professor at Yonsei University, South Korea. He was an Assistant Professor in Sungkyunkwan University, South Korea and Senior Lecturer in the Institute of Mathematical Sciences, University of Malaya, Malaysia. Presently, he is working as an Associate Professor with King Abdulaziz University, Saudi Arabia. He has published more than 100 articles in journals of repute. He is a member of editorial boards in 10 journals and a reviewer for more than 30 journals. His areas of interest include convective heat and mass transfer, CFD, nanofluids, micro-channel heat sinks, and porous media.

Bhuvaneswari Marimuthu received MSc, MPhil, and $\mathrm{PhD}$ degrees from Bharathiar University, Coimbatore, India. She received the Post-Doctoral Fellowship twice from National Cheng Kung University, Taiwan, and twice from Sungkyunkwan University, South Korea. Then, she worked as a research fellow at University of Malaya, Malaysia. Her areas of interest are numerical and analytical methods for PDE and heat and mass transfer.

Ali S. Alshomrani received $\mathrm{MSc}$ and $\mathrm{PhD}$ degrees from Heriot Watt University, UK. He is working as an Assistant Professor at King Abdulaziz University, Saudi Arabia. His areas of interest are numerical methods and dynamical systems.

Zailan Siri received the MSc and $\mathrm{PhD}$ degrees in Applied Mathematics from Univeristi Putra Malaysia and Universiti Kebangsaan Malaysia, respectively. Since 2012, he has been working as a Senior Lecturer in University of Malaya, Malaysia. His current research interests include fluid dynamics and numerical methods. 